\title{
Emergent two-Higgs doublet models
}

\author{
Tomohiro Abe ${ }^{a, b}$ and Yuji Omura ${ }^{b}$ \\ ${ }^{a}$ Institute for Advanced Research, Nagoya University, \\ Furo-cho Chikusa-ku, Nagoya, Aichi, 464-8602 Japan \\ ${ }^{b}$ Kobayashi-Maskawa Institute for the Origin of Particles and the Universe, Nagoya University, \\ Furo-cho Chikusa-ku, Nagoya, Aichi, 464-8602 Japan \\ E-mail: abetomo@kmi.nagoya-u.ac.jp, yujiomur@kmi.nagoya-u.ac.jp
}

ABSTRACT: We investigate origin of three features that are often assumed in analysis of two-Higgs doublet models: (i) softly broken $Z_{2}$ symmetry, (ii) CP invariant Higgs potential, and (iii) degenerated mass spectra. We extend electroweak gauge symmetry, introducing extra gauge symmetry and extra scalars, and we show that our models effectively derive two-Higgs doublet models at low energy which naturally hold the three features. We also find that the models can solve the strong CP problem.

Keywords: Beyond Standard Model, Higgs Physics

ARXIV EPRINT: 1606.06537 


\section{Contents}

1 Introduction 1

2 Review of two-Higgs doublet models $\quad 3$

3 Emergence of two-Higgs doublet models satisfying the three conditions 4

3.1 A model with extended electroweak gauge symmetry 5

3.2 Emergence of the type-I two-Higgs doublet model 6

3.3 Emergence of the inert doublet model 9

4 Extension of the model $\quad 10$

$\begin{array}{lll}4.1 & \text { Emergence of the type-III two-Higgs doublet model } & 10\end{array}$

4.2 Emergence of the type-II, -X, and -Y two Higgs doublet models with a discrete symmetry 11

4.3 Emergence of the type-II, -X, and -Y two Higgs doublet models with a global symmetry 12

$\begin{array}{lll}5 & \text { Summary } & 12\end{array}$

\section{Introduction}

The Standard Model (SM) is elaborately constructed and succeeds in reproducing almost all experimental results so far. The model is based on a $\mathrm{SU}(3)_{c} \times \mathrm{SU}(2)_{L} \times \mathrm{U}(1)_{Y}$ gauge theory, and the unification of the weak and electromagnetic interactions is achieved by introducing a Higgs doublet and spontaneous electroweak (EW) symmetry breaking. In 2012, the signal predicted by the SM Higgs was finally found at the LHC [1, 2], so that we are sure that the Higgs particle really exists in our nature.

On the other hand, we expect that the SM is an effective model and new physics behind the SM is also discovered near future. One reason is that the SM has several nontrivial structures. For instance, the anomaly-free conditions for the gauge symmetries are achieved by the very non-trivial charge assignment of the quarks and leptons. Besides, the Higgs couplings with the fermions are unnaturally hierarchical and we do not know the origin of the Higgs potential to trigger the EW symmetry breaking. We note that some deviations from the SM predictions in some experiments have been reported, so they also motivate new physics beyond the SM.

In fact, many extensions of the SM have been proposed so far. However, it is not so easy to consider the extensions, because of the stringent experimental constraints and the non-trivial structures of the SM. As we mentioned above, the anomaly-free conditions are satisfied miraculously, so that it is not simple to introduce new fermions charged under the 
SM gauge groups. Furthermore, the experimental constraints are getting stronger according to the precision measurements. Especially, the SM is very successful in flavor physics and we know that new physics should avoid tree-level Flavor Changing Neutral Currents (FCNCs).

Two-Higgs doublet model $(2 \mathrm{HDM})$ is one of the simple extended models without such serious problems. In this extension, one extra Higgs doublet is introduced, and the anomaly-free conditions are the same as the SM ones. In fact, $2 \mathrm{HDM}$ s have been widely discussed so far motivated by the experimental anomalies, such as the muon $g-2[3-15]$. Besides, many Beyond Standard Models (BSMs) to solve the theoretical issues in the SM predict such an extra Higgs doublet, so that 2HDMs have been well analyzed as the lowenergy effective models, although it is often difficult to derive $2 \mathrm{HDMs}$ effectively. This is because extra other matters generally reside at the same scale as the extra Higgs doublet in many BSMs.

Even in such a simple BSM, however, some assumptions are usually supposed to avoid the conflicts with the experimental results. For instance, there are generally tree-level FCNCs in 2HDMs, if the two Higgs doublets are not distinguishable. We usually assign a softly broken $Z_{2}$ symmetry to avoid the tree-level FCNCs, and then we only allow the minimal flavor violation (MFV) [16]. Moreover, the Higgs potential contains CP violating terms in general. This is an attractive point of $2 \mathrm{HDM}$, for example, for electroweak beryogenesis scenario. Nevertheless, CP invariance is often assumed in 2HDM. In addition, the results in the precision measurements of the EW interaction should be consistent with the predictions of $2 \mathrm{HDMs}$. For example, the EW precision tests suggest that $\rho$ parameter is close to one. This requires unnaturally degenerated dimensionless couplings, namely $\lambda_{4}=\lambda_{5}$, in 2HDMs.

These three assumptions are required by the experimental results. If there is an extra Higgs doublet in our nature, these three features would offer an important clue as to the unknown physics behind the SM. In this paper, we investigate a possible underlying theory which effectively derives the $2 \mathrm{HDM}$ respecting the three features at low energy. Especially, we study some models where the electroweak gauge symmetry is extended, ${ }^{1}$ and show that the models naturally have the three features often assumed. We note that $2 \mathrm{HDMs}$ with gauged U(1) symmetry have been proposed as the origin of the softly broken $Z_{2}$ [18-20]. This $U(1)$ symmetry may be originated from the grand unified theory [20], but the setup is complicated and the $\rho$ parameter is deviated from one at the tree level [18-20]. Compared to this proposal, our models in this paper are much simple and can avoid too large deviation of the $\rho$ parameter.

The rest of this paper is organized as follows. In section 2 , we briefly review the 2HDM. In section 3.1, we propose a model with extended electroweak gauge symmetry. In section 3.2, we derive the low energy effective theory of the model discussed in section 3.1, and show that it behaves as the type-I $2 \mathrm{HDM}$ with softly broken $Z_{2}$ symmetry without any $\mathrm{CP}$ phases in the Higgs potential. The model also predicts degenerated mass spectra for the CP-odd and the charged Higgs bosons. Thus all the three conditions are automatically satisfied. In section 4 , we extend the model to lead 2HDM other than the type-I 2HDM. Section 5 is devoted to our conclusion.

\footnotetext{
${ }^{1}$ Another possibility is to impose some symmetries on the Higgs potential [17].
} 


\section{Review of two-Higgs doublet models}

In this section, we review the two-Higgs doublet model with the softly broken $Z_{2}$ symmetry widely discussed. We have two Higgs fields, $\Phi_{1}$ and $\Phi_{2}$, charged under $\mathrm{SU}(2)_{L} \times \mathrm{U}(1)_{Y}$. In general the Higgs potential at the renormalizable level is given as follows:

$$
\begin{aligned}
V= & m_{1}^{2} \Phi_{1}^{\dagger} \Phi_{1}+m_{2}^{2} \Phi_{2}^{\dagger} \Phi_{2}-\left(m_{3}^{2} \Phi_{1}^{\dagger} \Phi_{2}+(\text { h.c. })\right) \\
& +\frac{1}{2} \lambda_{1}\left(\Phi_{1}^{\dagger} \Phi_{1}\right)^{2}+\frac{1}{2} \lambda_{2}\left(\Phi_{2}^{\dagger} \Phi_{2}\right)^{2}+\lambda_{3}\left(\Phi_{1}^{\dagger} \Phi_{1}\right)\left(\Phi_{2}^{\dagger} \Phi_{2}\right)+\lambda_{4}\left(\Phi_{1}^{\dagger} \Phi_{2}\right)\left(\Phi_{2}^{\dagger} \Phi_{1}\right) \\
& +\left(\frac{1}{2} \lambda_{5}\left(\Phi_{1}^{\dagger} \Phi_{2}\right)^{2}+\lambda_{6}\left(\Phi_{1}^{\dagger} \Phi_{1}\right)\left(\Phi_{1}^{\dagger} \Phi_{2}\right)+\lambda_{7}\left(\Phi_{2}^{\dagger} \Phi_{2}\right)\left(\Phi_{1}^{\dagger} \Phi_{2}\right)+(\text { h.c. })\right) .
\end{aligned}
$$

Four parameters, $m_{3}^{2}, \lambda_{5}, \lambda_{6}$, and $\lambda_{7}$, can be complex, and they are CP violating. Now, we impose a softly broken $Z_{2}$ symmetry to the Higgs fields: $\left(\Phi_{1}, \Phi_{2}\right) \rightarrow\left(\Phi_{1},-\Phi_{2}\right)$. The $Z_{2}$ symmetry forbids $\lambda_{6}$ and $\lambda_{7}$ terms. The $m_{3}^{2}$ term breaks the $Z_{2}$ symmetry softly, but can shift the scalar masses. Let us define the vacuum expectation values (VEVs) of the Higgs fields as

$$
\left\langle\Phi_{1}\right\rangle=\frac{v_{d}}{\sqrt{2}},\left\langle\Phi_{2}\right\rangle=\frac{v_{u}}{\sqrt{2}},
$$

and then the relation with the Fermi constant is

$$
v_{u}^{2}+v_{d}^{2}=\frac{1}{\sqrt{2} G_{F}} \equiv v^{2} \simeq(246 \mathrm{GeV})^{2} .
$$

We also define $\beta$ as follows:

$$
\cos \beta=\frac{v_{d}}{v}, \sin \beta=\frac{v_{u}}{v} .
$$

We have eight degrees of freedom in the scalar fields and three of them are eaten by the gauge bosons, and thus we have five physical states: two of them are CP-even states $\left(h, H^{0}\right)$, one is a CP-odd state $\left(A^{0}\right)$, and the others are a pair of charged scalar $\left(H^{ \pm}\right)$. Their masses are given by the parameters in the Higgs potential. When the $Z_{2}$ symmetry is imposed, the charged and CP-odd Higgs masses satisfy

$$
\begin{aligned}
m_{A}^{2} & =M^{2}-\lambda_{5} v^{2}, \\
m_{H^{ \pm}}^{2} & =m_{A}^{2}+\frac{\lambda_{5}-\lambda_{4}}{2} v^{2},
\end{aligned}
$$

where

$$
M^{2}=\frac{m_{3}^{2}}{\sin \beta \cos \beta} .
$$

Here $\lambda_{5}$ is assumed to be real.

There are four different assignments of the $Z_{2}$ symmetry to fermions [21-23]. The assignments and the model names are summarized in table 1 . These assignments forbid FCNCs involving neutral scalars. The physics in the each 2HDM has been widely studied, although their origins of $Z_{2}$ symmetries are unclear.

In $2 \mathrm{HDMs}$, the constraint of the $\rho$ parameter, or $T$ parameter, is important. It depends on the scalar masses and $W W h$-coupling $\left(g_{W W h}=\kappa_{V} g_{W W h}^{S M}\right)$. Since the LHC result implies 


\begin{tabular}{|c|cc|cc|ccc|}
\hline & $\Phi_{1}$ & $\Phi_{2}$ & $q_{L}$ & $\ell_{L}$ & $u_{R}$ & $d_{R}$ & $\ell_{R}$ \\
\hline type-I & + & - & + & + & - & - & - \\
type-II & + & - & + & + & - & + & + \\
type-X & + & - & + & + & - & - & + \\
type-Y & + & - & + & + & - & + & - \\
\hline type-III & \multicolumn{1}{|c|}{ no $Z_{2}$ assingment } \\
\hline
\end{tabular}

Table 1. $Z_{2}$ assingment of the matters in 2HDM. If the model not assign the $Z_{2}$ symmetry is called type-III.

that the coupling is almost the same as the SM prediction, we assume $\kappa_{V} \simeq 1$. Then the $T$ parameter is approximately evaluated as

$$
\begin{array}{r}
\alpha T \simeq \frac{1}{16 \pi^{2} v^{2}}\left(m_{H^{0}}^{2}+\frac{m_{A}^{4}\left(m_{H^{ \pm}}^{2}-m_{H^{0}}^{2}\right)}{\left(m_{A}^{2}-m_{H^{ \pm}}^{2}\right)\left(m_{A}^{2}-m_{H^{0}}^{2}\right)} \ln \frac{m_{H^{ \pm}}^{2}}{m_{A}^{2}}\right. \\
\left.-\frac{m_{H^{0}}^{4}\left(m_{H^{ \pm}}^{2}-m_{A}^{2}\right)}{\left(m_{H^{0}}^{2}-m_{H^{ \pm}}^{2}\right)\left(m_{A}^{2}-m_{H^{0}}^{2}\right)} \ln \frac{m_{H^{ \pm}}^{2}}{m_{H^{0}}^{2}}\right) .
\end{array}
$$

We easily find that the right-hand side is vanishing when either $m_{A}=m_{H^{ \pm}}$or $m_{H^{0}}=m_{H^{ \pm}}$ is satisfied [24]. Since the right-hand side should be less than $\mathcal{O}\left(10^{-3}\right)$ according to the electroweak precision measurements [25], two of the masses should be highly degenerated. This degeneracy requires a parameter tuning in the Higgs potential. For example $\lambda_{4} \simeq \lambda_{5}$ is required for $m_{A} \simeq m_{H^{ \pm}}$. It is unclear whether a mechanism which makes $\lambda_{4} \simeq \lambda_{5}$ exists or not within the $2 \mathrm{HDM}$.

\section{Emergence of two-Higgs doublet models satisfying the three conditions}

In this section, we consider the extension of the SM that leads 2HDMs at low energy. Our effective $2 \mathrm{HDM}$ will satisfy the following three conditions to evade the stringent experimental constraints:

(i) softly broken $Z_{2}$ symmetry is remained,

(ii) Higgs potential is CP invariant,

(iii) Two scalars are degenerate.

At first, we consider a model which effectively realizes the type-I 2HDM. Note that only one Higgs doublet couples with all fermions and the other Higgs doublet does not in the type-I 2HDM. Depending on the vacuum alignment of the Higgs fields, the model can also leads to the Inert 2HDM [26, 27], where the lightest neutral scalar becomes stable and a dark matter candidate. 


\begin{tabular}{|c|ccc|cc|ccc|}
\hline & $H_{1}$ & $H_{2}$ & $\phi_{3}$ & $q_{L}^{i}$ & $\ell_{L}^{i}$ & $u_{R}^{i}$ & $d_{R}^{i}$ & $e_{R}^{i}$ \\
\hline $\mathrm{SU}(2)_{0}$ & $\mathbf{2}$ & $\mathbf{1}$ & $\mathbf{2}$ & $\mathbf{2}$ & $\mathbf{2}$ & $\mathbf{1}$ & $\mathbf{1}$ & $\mathbf{1}$ \\
$\mathrm{SU}(2)_{1}$ & $\mathbf{2}$ & $\mathbf{2}$ & $\mathbf{1}$ & $\mathbf{1}$ & $\mathbf{1}$ & $\mathbf{1}$ & $\mathbf{1}$ & $\mathbf{1}$ \\
$\mathrm{U}(1)_{2}$ & 0 & $\frac{1}{2}$ & $\frac{1}{2}$ & $\frac{1}{6}$ & $-\frac{1}{2}$ & $\frac{2}{3}$ & $-\frac{1}{3}$ & -1 \\
$\mathrm{SU}(3)_{c}$ & $\mathbf{1}$ & $\mathbf{1}$ & $\mathbf{1}$ & $\mathbf{3}$ & $\mathbf{1}$ & $\mathbf{3}$ & $\mathbf{3}$ & $\mathbf{1}$ \\
\hline
\end{tabular}

Table 2. Quantum numbers of the Higgs and matter fields $(i=1,2,3)$.

\subsection{A model with extended electroweak gauge symmetry}

Now, we consider a BSM with extended gauge symmetry, based on ref. [28]. In this model, the electroweak gauge symmetry is extended to $\mathrm{SU}(2)_{0} \times \mathrm{SU}(2)_{1} \times \mathrm{U}(1)_{2}$. We introduce three scalar fields, $H_{1}, H_{2}$ and $\phi_{3}$, for the gauge symmetry breaking. Their quantum numbers are summarized in table 2. $q_{L}^{i}, u_{R}^{i}$ and $d_{R}^{i}$ denote left-handed and right-handed quarks respectively. $\ell_{L}^{i}$ and $e_{R}^{i}$ are left-handed and right-handed leptons. The left-handed matter fields are charged under the $\mathrm{SU}(2)_{0}$ gauge symmetry.

The gauge symmetric Yukawa couplings, which generate the mass matrices for the matters, are given by

$$
\mathcal{L}^{\text {Yukawa }}=-\sum_{i, j} y_{u}^{i j} \overline{q_{L}^{i}} \widetilde{\phi_{3}} u_{R}^{j}-\sum_{i, j} y_{d}^{i j} \overline{q_{L}^{i}} \phi_{3} d_{R}^{j}-\sum_{i, j} y_{e}^{i j} \overline{\ell_{L}^{i}} \phi_{3} e_{R}^{j}+\text { h.c. }
$$

The scalar potential is written down at the renormalizable level:

$$
\begin{aligned}
V\left(H_{1}, H_{2}, \phi_{3}\right)= & \mu_{1}^{2} \operatorname{tr}\left(H_{1} H_{1}^{\dagger}\right)+\mu_{2}^{2} H_{2}^{\dagger} H_{2}+\mu_{3}^{2} \phi_{3}^{\dagger} \phi_{3} \\
& +\frac{1}{2}\left(\kappa \phi_{3}^{\dagger} H_{1} H_{2}+(\text { h.c. })\right) \\
& +\widetilde{\lambda}_{1}\left(\operatorname{tr}\left(H_{1} H_{1}^{\dagger}\right)\right)^{2}+\widetilde{\lambda}_{2}\left(H_{2}^{\dagger} H_{2}\right)^{2}+\widetilde{\lambda}_{3}\left(\phi_{3}^{\dagger} \phi_{3}\right)^{2} \\
& +\widetilde{\lambda}_{12} \operatorname{tr}\left(H_{1} H_{1}^{\dagger}\right)\left(H_{2}^{\dagger} H_{2}\right)+\widetilde{\lambda}_{23}\left(H_{2}^{\dagger} H_{2}\right)\left(\phi_{3}^{\dagger} \phi_{3}\right)+\widetilde{\lambda}_{31}\left(\phi_{3}^{\dagger} \phi_{3}\right) \operatorname{tr}\left(H_{1} H_{1}^{\dagger}\right),
\end{aligned}
$$

Note that $H_{1}$ is a two by two matrix charged under $\mathrm{SU}(2)_{0} \times \mathrm{SU}(2)_{1}$ and is defined as the field to satisfy

$$
\tau^{2} H_{1}^{*} \tau^{2}=H_{1}
$$

where $\tau^{2}$ is the second Pauli matrix. The Higgs potential contains one complex parameter $\kappa$, but its phase are eliminated by a field redefinition of $H_{2}$. Therefore we can take all the parameters in the Higgs potential as real numbers. We can also take the VEVs of the Higgs fields as real thanks to the gauge symmetries. Hence we do not have any source of the $\mathrm{CP}$ violation in the Higgs sector.

When the heavy gauge bosons are extremely heavy, we can integrate them out and construct a low-energy effective models. The effective model is similar to the two-Higgs doublet models with softly broken $Z_{2}$ symmetry. In the followings, we discuss the effective model and see that it can be interpreted as the type-I 2HDM or the Inert 2HDM satisfying our three conditions, (i)-(iii). 


\subsection{Emergence of the type-I two-Higgs doublet model}

Here, we discuss our effective model predicted by the BSM with the extended gauge symmetry. There are two $\mathrm{SU}(2)$ gauge symmetries, $\mathrm{SU}(2)_{0} \times \mathrm{SU}(2)_{1}$, and one scalar, $H_{1}$, charged under the both symmetries. Then, the VEV of $H_{1}$ breaks down the symmetries to $\mathrm{SU}(2)_{L}$ :

$$
\mathrm{SU}(2)_{0} \times \mathrm{SU}(2)_{1} \rightarrow \mathrm{SU}(2)_{L}
$$

We parametrize $H_{1}$,

$$
H_{1}=\frac{1}{2}\left(v_{1}+h_{1}\right) U_{1}, \text { where } U_{1}=\exp \left(i \frac{\tau^{a} \pi_{1}^{a}}{v_{1}}\right) .
$$

Here $v_{1}$ is the VEV of $H_{1}$. We choose appropriate parameters in the Higgs potential to realize non-zero VEVs. According to the symmetry breaking, Nambu-Goldstone (NG) bosons appear and are eaten by the $\mathrm{SU}(2)$ symmetry orthogonal to $\mathrm{SU}(2)_{L}$.

We define the gauge fields for $\mathrm{SU}(2)_{0} \times \mathrm{SU}(2)_{1} \times \mathrm{U}(1)_{2}$ as

$$
\mathcal{L}^{\text {gauge }}=-\frac{1}{4} \sum_{a=1}^{3} W_{0 \mu \nu}^{a} W_{0}^{a \mu \nu}-\frac{1}{4} \sum_{a=1}^{3} W_{1 \mu \nu}^{a} W_{1}^{a \mu \nu}-\frac{1}{4} B_{\mu \nu} B^{\mu \nu},
$$

where the field strengths are depicted by the gauge fields, $W_{0 \mu}, W_{1 \mu}$ and $B_{\mu}$ of $\mathrm{SU}(2)_{0} \times \mathrm{SU}(2)_{1} \times \mathrm{U}(1)_{2}: W_{0 \mu \nu}=\partial_{\mu} W_{0 \nu}-\partial_{\nu} W_{0 \mu}+i g_{0}\left[W_{0 \mu}, W_{0 \nu}\right]$, and $W_{1 \mu \nu}=\partial_{\mu} W_{1 \nu}-$ $\partial_{\nu} W_{1 \mu}+i g_{1}\left[W_{1 \mu}, W_{1 \nu}\right]$, and $B_{\mu \nu}=\partial_{\mu} B_{\nu}-\partial_{\nu} B_{\mu}$, respectively.

The nonzero VEV of $H_{1}$ generates the mass term of the broken gauge symmetry, which is a linear combination of $W_{0 \mu}$ and $W_{1 \mu}$, and $\pi_{1}^{a}$ in $U_{1}$ are eaten by the gauge field.

For convenience, let us redefine the gauge field, $W_{1}^{\mu}$, as

$$
V_{1}^{\mu}=U_{1} W_{1}^{\mu} U_{1}^{\dagger}+\frac{1}{i g_{1}} U_{1} \partial_{\mu} U_{1}^{\dagger}
$$

$V_{1}^{\mu}$ is transformed by the $\mathrm{SU}(2)_{0}$ gauge symmetry with the gauge coupling of $\mathrm{SU}(2)_{1}$, depicted by $g_{1}$. It is useful to use $V_{1}^{\mu}$ instead of $W_{1}^{\mu}$ in this analysis. Then, we take the linear combinations of $W_{0}^{\mu}$ and $V_{1}^{\mu}$ :

$$
\begin{aligned}
\rho_{\mu} & =c W_{0}^{\mu}-s V_{1}^{\mu}, \\
W_{\mu} & =s W_{0}^{\mu}+c V_{1}^{\mu},
\end{aligned}
$$

where the mixing is given by

$$
c=\frac{g_{0}}{\sqrt{g_{0}^{2}+g_{1}^{2}}}, \quad s=\frac{g_{1}}{\sqrt{g_{0}^{2}+g_{1}^{2}}} .
$$

In the effective model, the gauge symmetry is $\mathrm{SU}(2)_{L} \times \mathrm{U}(1)_{Y}$, and $W_{\mu}$ is the gauge boson of $\mathrm{SU}(2)_{L}$, which is given by $\mathrm{SU}(2)_{0}$ with a different gauge coupling. Note that $\rho^{\mu}$ is a massive $\mathrm{SU}(2)_{L}$ triplet. Let us define the gauge couplings in the effective model:

$$
g=\frac{g_{0} g_{1}}{\sqrt{g_{0}^{2}+g_{1}^{2}}}, \quad g_{\rho}=\sqrt{g_{0}^{2}+g_{1}^{2}}
$$


where $g$ is the $\mathrm{SU}(2)_{L}$ gauge coupling associated with $W_{\mu}$. Based on the above definitions, we find the correspondence between the fields in the original and in the effective:

$$
\begin{aligned}
W_{1}^{\mu \nu} & =U_{1} V_{1}^{\mu \nu} U_{1}^{\dagger}, \\
W_{0}^{\mu \nu} & =s W^{\mu \nu}+c\left(D^{\mu} \rho^{\nu}-D^{\nu} \rho^{\mu}\right)+i g_{0} c^{2}\left[\rho^{\mu}, \rho^{\nu}\right], \\
V_{1}^{\mu \nu} & =c W^{\mu \nu}-s\left(D^{\mu} \rho^{\nu}-D^{\nu} \rho^{\mu}\right)+i g_{1} s^{2}\left[\rho^{\mu}, \rho^{\nu}\right], \\
\hat{D}_{\mu} H_{2} & =U_{1}^{\dagger}\left(D_{\mu} \phi_{2}-i g_{1} s \rho_{\mu} \phi_{2}\right), \\
\hat{D}_{\mu} \phi_{3} & =D_{\mu} \phi_{3}+i g_{0} c \rho_{\mu} \phi_{3}, \\
\hat{D}_{\mu} \psi_{L} & =D_{\mu} \psi_{L}+i g_{0} c \rho_{\mu} \psi_{L} .
\end{aligned}
$$

Here, $\phi_{2}$ is defined as

$$
\phi_{2}=U_{1} H_{2},
$$

$\hat{D}_{\mu}$ is the covariant derivative with respect to $\mathrm{SU}(2)_{0} \times \mathrm{SU}(2)_{1} \times \mathrm{U}(1)_{2}$, and $D_{\mu}$ is the covariant derivative with respect to $\mathrm{SU}(2)_{L} \times \mathrm{U}(1)_{2}$. Namely, $D_{\mu}$ contains only $W_{\mu}$ and $B_{\mu}$ :

$$
\begin{aligned}
D_{\mu} \psi & =\left(\partial_{\mu}+i g W_{\mu}+i g_{2} Y B_{\mu}\right) \psi, \\
D_{\mu} \rho_{\nu} & =\partial_{\mu} \rho_{\nu}+i g\left[W_{\mu}, \rho_{\nu}\right] .
\end{aligned}
$$

$\psi_{L}$ is the left-handed $\mathrm{SM}$ fermions charged under $\mathrm{SU}(2)_{0}\left(\mathrm{SU}(2)_{L}\right) . \psi_{R}$ is the right-handed.

Finally, we find our effective Lagrangian as follows,

$$
\mathcal{L}=\mathcal{L}^{(0)}+\mathcal{L}^{(1)}+\mathcal{L}^{(2)}-V\left(h_{1}, \phi_{2}, \phi_{3}\right),
$$

where $\mathcal{L}^{(0)}, \mathcal{L}^{(1)}$, and $\mathcal{L}^{(2)}$ are given by

$$
\begin{aligned}
\mathcal{L}^{(0)}= & +\bar{\psi}_{L} i \gamma^{\mu} D_{\mu} \psi_{L}+\bar{\psi}_{R} i \gamma^{\mu} D_{\mu} \psi_{R}-\bar{\psi}_{L} \tilde{\phi}_{3} y_{u} \psi_{R}-\bar{\psi}_{L} \phi_{3} y_{d} \psi_{R}+(h . c .) \\
& +\frac{1}{2} \partial_{\mu} h_{1} \partial^{\mu} h_{1}+D_{\mu} \phi_{2}^{\dagger} D_{\mu} \phi_{2}+D_{\mu} \phi_{3}^{\dagger} D_{\mu} \phi_{3} \\
& -\frac{1}{2} \operatorname{tr}\left(W_{\mu \nu} W^{\mu \nu}\right)-\frac{1}{4} B_{\mu \nu} B^{\mu \nu} \\
\mathcal{L}^{(1)}= & -g_{0} c \bar{\psi}_{L} \gamma^{\mu} \rho_{\mu} \psi_{L} \\
& +i g_{1} s\left(\phi_{2}^{\dagger} \rho_{\mu} D^{\mu} \phi_{2}-\left(D^{\mu} \phi_{2}^{\dagger}\right) \rho_{\mu} \phi_{2}\right) \\
& -i g_{0} c\left(\phi_{3}^{\dagger} \rho_{\mu} D^{\mu} \phi_{3}-\left(D^{\mu} \phi_{3}^{\dagger}\right) \rho_{\mu} \phi_{3}\right) \\
\mathcal{L}^{(2)}= & +\frac{1}{4} \rho_{\mu}^{a} \rho^{a \mu}\left(g_{1}^{2} s^{2} \phi_{2}^{\dagger} \phi_{2}+g_{0}^{2} c^{2} \phi_{3}^{\dagger} \phi_{3}\right)+\frac{1}{8}\left(v_{1}+h_{1}\right)^{2} g_{\rho}^{2} \rho_{\mu}^{a} \rho^{a \mu} \\
& -i g \operatorname{tr}\left(\left[\rho_{\mu}, \rho_{\nu}\right] W^{\mu \nu}\right) \\
& -\frac{1}{2} \operatorname{tr}\left(\left(D_{\mu} \rho_{\nu}-D_{\nu} \rho_{\mu}\right)\left(D^{\mu} \rho^{\nu}-D^{\nu} \rho^{\mu}\right)\right) \\
& -i\left(g_{0} c^{3}+g_{1} s^{3}\right) \operatorname{tr}\left(\left[\rho_{\mu}, \rho_{\nu}\right]\left(D^{\mu} \rho^{\nu}-D^{\nu} \rho^{\mu}\right)\right) \\
& +\frac{1}{2}\left(g_{0}^{2} c^{4}+g_{1}^{2} s^{4}\right) \operatorname{tr}\left(\left[\rho_{\mu}, \rho_{\nu}\right]\left[\rho^{\mu}, \rho^{\nu}\right]\right) .
\end{aligned}
$$

$V\left(h_{1}, \phi_{2}, \phi_{3}\right)$ is the scalar potential for the effective lagrangian, given by substituting the scalar fields to eq. (3.2). Now, $h_{1}$ is gauge singlet, and $\phi_{2}$ and $\phi_{3}$ are $\mathrm{SU}(2)_{L}$-doublet. 
Then, we successfully derive a $2 \mathrm{HDM}$ with an extra singlet scalar boson and an extra $\mathrm{SU}(2)_{L}$ vector boson. Note that $\mathrm{U}(1)_{2}$ is identified to $\mathrm{U}(1)_{Y}$ in the SM.

Now, we assume that the breaking scale of $\mathrm{SU}(2)_{1} \times \mathrm{SU}(2)_{2}$ is much higher than the EW scale. We find that $\rho^{\mu}$ and $h_{1}$ gain the mass proportional to $v_{1}$ so that they become extremely heavy and can be integrated out much above the EW scale under this assumption. Then the effective lagrangian around the EW scale can be described as

$$
\left.\mathcal{L}=\mathcal{L}^{(0)}+V_{\text {eff }}\left(\phi_{2}, \phi_{3}\right)+\text { (higher dimensional operators }\right) .
$$

Defining the VEVs of $\phi_{2}$ and $\phi_{3}$ as $v_{2} / \sqrt{2}$ and $v_{3} / \sqrt{2}, V_{\text {eff }}\left(\phi_{2}, \phi_{3}\right)$ is expressed as below:

$$
\begin{aligned}
V_{\mathrm{eff}}\left(\phi_{2}, \phi_{3}\right)= & -\kappa \frac{v_{3} v_{1}}{4 v_{2}}\left(\phi_{2}^{\dagger} \phi_{2}-\frac{v_{2}^{2}}{2}\right)-\kappa \frac{v_{1} v_{2}}{4 v_{3}}\left(\phi_{3}^{\dagger} \phi_{3}-\frac{v_{3}^{2}}{2}\right)+\kappa \frac{v_{1}}{4}\left(\phi_{2}^{\dagger} \phi_{3}+\phi_{3}^{\dagger} \phi_{2}\right) \\
& +\left(\tilde{\lambda}_{2}+\frac{v_{1}^{2} \tilde{\lambda}_{12}^{2}}{2 m_{h_{1}}^{2}}\right)\left(\phi_{2}^{\dagger} \phi_{2}-\frac{v_{2}^{2}}{2}\right)^{2}+\left(\tilde{\lambda}_{3}+\frac{v_{1}^{2} \tilde{\lambda}_{31}^{2}}{2 m_{h_{1}}^{2}}\right)\left(\phi_{3}^{\dagger} \phi_{3}-\frac{v_{3}^{2}}{2}\right)^{2} \\
& +\left(\tilde{\lambda}_{23}+\frac{v_{1}^{2}}{m_{h_{1}}^{2}} \tilde{\lambda}_{12} \tilde{\lambda}_{31}\right)\left(\phi_{2}^{\dagger} \phi_{2}-\frac{v_{2}^{2}}{2}\right)\left(\phi_{3}^{\dagger} \phi_{3}-\frac{v_{3}^{2}}{2}\right) \\
& +\frac{\kappa^{2}}{32 m_{h_{1}}^{2}}\left(\phi_{2}^{\dagger} \phi_{3}+\phi_{3}^{\dagger} \phi_{2}-v_{2} v_{3}\right)^{2} \\
& +\frac{\kappa v_{1}}{4 m_{h_{1}}^{2}} \tilde{\lambda}_{12}\left(\phi_{2}^{\dagger} \phi_{2}-\frac{v_{2}^{2}}{2}\right)\left(\phi_{2}^{\dagger} \phi_{3}+\phi_{3}^{\dagger} \phi_{2}-v_{2} v_{3}\right) \\
& +\frac{\kappa v_{1}}{4 m_{h_{1}}^{2}} \tilde{\lambda}_{31}\left(\phi_{3}^{\dagger} \phi_{3}-\frac{v_{3}^{2}}{2}\right)\left(\phi_{2}^{\dagger} \phi_{3}+\phi_{3}^{\dagger} \phi_{2}-v_{2} v_{3}\right) .
\end{aligned}
$$

We can easily find out the correspondence between the Higgs potential in eq. (2.1) and $V_{\text {eff }}\left(\phi_{2}, \phi_{3}\right)$, according to the following identifications,

$$
\begin{array}{ll}
\phi_{2}=\Phi_{1}, & v_{2}=v_{d}, \\
\phi_{3}=\Phi_{2}, & v_{3}=v_{u} .
\end{array}
$$

The each parameter in $V_{\text {eff }}$ corresponds to the one in eq. (2.1) as follows:

$$
\begin{aligned}
& m_{1}^{2}=-\frac{\kappa v_{1} v_{3}}{4 v_{2}}-v_{2}^{2}\left(\tilde{\lambda}_{2}+\frac{v_{1}^{2} \tilde{\lambda}_{12}^{2}}{2 m_{h_{1}}^{2}}\right)-\frac{v_{3}^{2}}{2}\left(\tilde{\lambda}_{23}+\frac{v_{1}^{2} \tilde{\lambda}_{12} \tilde{\lambda}_{31}}{m_{h_{1}}^{2}}\right)-\frac{\kappa v_{1} v_{2} v_{3}}{4 m_{h_{1}}^{2}} \tilde{\lambda}_{12} \\
& m_{2}^{2}=-\frac{\kappa v_{1} v_{2}}{4 v_{3}}-v_{3}^{2}\left(\tilde{\lambda}_{3}+\frac{v_{1}^{2} \tilde{\lambda}_{31}^{2}}{2 m_{h_{1}}^{2}}\right)-\frac{v_{2}^{2}}{2}\left(\tilde{\lambda}_{23}+\frac{v_{1}^{2} \tilde{\lambda}_{12} \tilde{\lambda}_{31}}{m_{h_{1}}^{2}}\right)-\frac{\kappa v_{1} v_{2} v_{3}}{4 m_{h_{1}}^{2}} \tilde{\lambda}_{12} \\
& m_{3}^{2}=\frac{\kappa v_{1}}{4}-\frac{\kappa^{2} v_{2} v_{3}}{16 m_{h_{1}}^{2}}-\frac{\kappa v_{1} v_{2}^{2}}{8 m_{h_{1}}^{2}} \tilde{\lambda}_{12}-\frac{\kappa v_{1} v_{3}^{2}}{8 m_{h_{1}}^{2}} \tilde{\lambda}_{31} \\
& \lambda_{1}=2 \tilde{\lambda}_{2}+\frac{v_{1}^{2} \tilde{\lambda}_{12}^{2}}{m_{h_{1}}^{2}} \\
& \lambda_{2}=2 \tilde{\lambda}_{3}+\frac{v_{1}^{2} \tilde{\lambda}_{31}^{2}}{m_{h_{1}}^{2}}
\end{aligned}
$$




$$
\begin{aligned}
\lambda_{3} & =\tilde{\lambda}_{23}+\frac{v_{1}^{2}}{m_{h_{1}}^{2}} \tilde{\lambda}_{12} \tilde{\lambda}_{31} \\
\lambda_{4}=\lambda_{5} & =\frac{\kappa^{2}}{16 m_{h_{1}}^{2}} \simeq 0 \\
\lambda_{6} & =\frac{\kappa v_{1}}{4 m_{h_{1}}^{2}} \tilde{\lambda}_{12} \simeq 0 \\
\lambda_{7} & =\frac{\kappa v_{1}}{4 m_{h_{1}}^{2}} \tilde{\lambda}_{31} \simeq 0
\end{aligned}
$$

The relation, $\lambda_{4}=\lambda_{5}$, is respected, so that $m_{H^{ \pm}}^{2}=m_{A}^{2}$ is satisfied, according to eq. (2.6). Since $m_{h_{1}}^{2}$ is $\mathcal{O}\left(v_{1}^{2}\right)$ and much heavier than the other dimensional parameters, we can conclude that $\lambda_{4}$ and $\lambda_{5}$ are very tiny. Similarly, $\lambda_{6}$ and $\lambda_{7}$ are almost vanishing, in our model. Therefore $V_{\text {eff }}$ is the same as the Higgs potential in $2 \mathrm{HDM}$ with softly broken $Z_{2}$ symmetry, keeping CP symmetry. Besides, $V_{\text {eff }}$ leads the degenerated masses for the CPodd and the charged Higgs bosons. Then we conclude that our model naturally explains the origins of the three conditions, (i)-(iii), that are often assumed in studies of $2 \mathrm{HDMs}^{2}$

\subsection{Emergence of the inert doublet model}

In this section, we consider one specific scenario, in the framework of the Type-I 2HDM. In general, the two Higgs doublets gain non-vanishing VEVs, but, in fact, $\phi_{2}$ need not develop a nonzero VEV because $\phi_{2}$ does not couple with the SM fermions. We focus on the scenario with $v_{2}=0$ below.

This kind of model is called the Inert $2 \mathrm{HDM}[26,27]$. This model predicts a stable neutral particle which can be a dark matter candidate. This is an attractive feature of this model. $v_{2}=0$ is realized by $\kappa=0$, as we see from eq. (3.2). Then, we write down the Higgs potential in this scenario:

$$
\begin{aligned}
V\left(h_{1}, \phi_{2}, \phi_{3}\right)= & \tilde{\lambda}_{3}\left(\phi_{3}^{\dagger} \phi_{3}-\frac{v_{3}^{2}}{2}\right)^{2} \\
& +\left(\mu_{2}^{2}+\frac{1}{2} \tilde{\lambda}_{12} v_{1}^{2}\right) \phi_{2}^{\dagger} \phi_{2}+\tilde{\lambda}_{2}\left(\phi_{2}^{\dagger} \phi_{2}\right)^{2}+\tilde{\lambda}_{23}\left(\phi_{2}^{\dagger} \phi_{2}\right)\left(\phi_{3}^{\dagger} \phi_{3}\right) \\
& -\frac{1}{4} \tilde{\lambda}_{31} v_{3}^{2} h_{1}^{2}+\frac{1}{4} \tilde{\lambda}_{1}\left(h_{1}^{2}+2 v_{1} h_{1}\right)^{2} \\
& +\frac{1}{2}\left(h_{1}^{2}+2 v_{1} h_{1}\right)\left(\tilde{\lambda}_{12} \phi_{2}^{\dagger} \phi_{2}+\frac{1}{2} \tilde{\lambda}_{31} \phi_{3}^{\dagger} \phi_{3}\right) .
\end{aligned}
$$

Even if $h_{1}$ is integrated out, $\lambda_{4}$ and $\lambda_{5}$ cannot be induced, as we see in eq. (3.34). Thus $\lambda_{4}=\lambda_{5}=0$ is satisfied at the tree level and this leads degenerate masses for the extra scalars of $\mathrm{H}_{2}$.

It is known that the degenerated CP-even and -odd neutral scalars enhance the cross section of the direct search for dark matters via the inelastic scattering of the neutral particles with nucleus through $Z$-boson exchanging [27]. This enhancement is enough large to exclude models. Then we have to find ways to split the masses of the two neutral scalars.

\footnotetext{
${ }^{2}$ Our model leads $m_{H^{ \pm}}=m_{A}^{2}$. We need other models in order to lead $m_{H^{ \pm}}=m_{H^{0}}^{2}$. Phenomenology with a light pseudoscalar in the latter condition is discussed, for example, in ref. [29].
} 


\begin{tabular}{|c|cc|cc|}
\hline & $Q_{L}$ & $Q_{R}$ & $L_{L}$ & $L_{R}$ \\
\hline $\mathrm{SU}(2)_{0}$ & $\mathbf{1}$ & $\mathbf{1}$ & $\mathbf{1}$ & $\mathbf{1}$ \\
$\mathrm{SU}(2)_{1}$ & $\mathbf{2}$ & $\mathbf{2}$ & $\mathbf{2}$ & $\mathbf{2}$ \\
$\mathrm{U}(1)_{2}$ & $\frac{1}{6}$ & $\frac{1}{6}$ & $-\frac{1}{2}$ & $-\frac{1}{2}$ \\
$\mathrm{SU}(3)_{c}$ & $\mathbf{3}$ & $\mathbf{3}$ & $\mathbf{1}$ & $\mathbf{1}$ \\
\hline
\end{tabular}

Table 3. Quantum numbers of the new fermion fields.

Naive expectation is that loop corrections split the masses. However, this does not happen due to an accidental U(1) symmetry. The original Lagrangian without the $\kappa$ term has an accidental symmetry under which $H_{2}$ transforms as $H_{2} \rightarrow \exp \left(i \alpha_{2}\right) H_{2}$ and all the other fields do not transform. This symmetry remains even at the low energy as a global $\mathrm{U}(1)$ symmetry only for $\phi_{2}$. This global symmetry allows $\lambda_{4}$ term, and we can expect the charged scalar mass is different from the other neutral scalar masses at loop level. On the other hand, this global symmetry forbids the $\lambda_{5}$ term, and thus the masses of the two neutral scalars keep degenerated even at loop level. Therefore our Inert 2HDM is excluded by the direct search for dark matters, if the relic abundance of dark matter is dominated by the neutral components of $\phi_{2}$.

\section{Extension of the model}

In the previous section, we have discussed BSMs with extended gauge symmetry which induce the type-I and the Inert 2HDMs as low-energy effective models. Based on the above discussion, we try to construct a model that leads other types of the 2HDMs at low energy.

In other types, not one but two Higgs doublet fields couple with the SM fermions, so that we cannot easily extend the gauge symmetry, under which the Higgs doublets are charged. We need some modifications of our model with $\mathrm{SU}(2)_{0} \times \mathrm{SU}(2)_{1} \times \mathrm{U}(1)_{2}$. In this section, we discuss some example ways to modify and extend our model.

\subsection{Emergence of the type-III two-Higgs doublet model}

For the type-II, -X, -Y, and -III two-Higgs doublet models, we need more than one Yukawa interaction terms. Then, not only $\phi_{3}$ but also $H_{1}$ and $H_{2}$ should be involved in the Yukawa interactions with the SM fermions. That is achieved if we allow the dimension-5 operators, $\bar{\psi}_{L} H_{1} H_{2} \psi_{R}$. This dimension-5 operators are generated if we add new vector-like fermions charged under $\mathrm{SU}(2)_{1}$ gauge symmetry as shown in table $3 .^{3}$ Using these new fermions

\footnotetext{
${ }^{3}$ This setup is similar to the models discussed in refs. [30, 31]. In those papers, the third generation in the quark sector is distinguished from the other generations.
} 
and the original fermions, the Yukawa interaction terms are given by

$$
\begin{aligned}
\mathcal{L}^{\text {Yukawa }}= & -\sum_{i, j} \bar{q}_{L}^{i} \tilde{\phi}_{3} y_{u}^{i j} u_{R}^{j}-\sum_{i, j} \bar{q}_{L}^{i} \phi_{3} y_{d}^{i j} d_{R}^{j}-\sum_{i} \bar{\ell}_{L}^{i} \phi_{3} y_{e}^{i} e_{R}^{i} \\
& -\sum_{i, j} \bar{q}_{L}^{i} H_{1} Y_{Q 1}^{i j} Q_{R}^{j}-\sum_{i, j} \bar{Q}_{R}^{i} M_{Q}^{i j} Q_{L}^{j}-\sum_{i, j} \bar{Q}_{L}^{i} \tilde{\phi}_{2} Y_{u 2}^{i j} u_{R}^{j}-\sum_{i, j} \bar{Q}_{L}^{i} \phi_{2} Y_{d 2}^{i j} d_{R}^{j} \\
& -\sum_{i, j} \bar{\ell}_{L}^{i} H_{1} Y_{L 1}^{i j} Q_{R}^{j}-\sum_{i, j} \bar{L}_{R}^{i} M_{L}^{i j} L_{L}^{j}-\sum_{i, j} \bar{L}_{L}^{i} \phi_{2} Y_{2 e}^{i j} e_{R}^{j} \\
& +(h . c .) .
\end{aligned}
$$

We can take $M_{Q}$ and $M_{L}$ as diagonal matrices without lose of generality by the transformation of the vector-like fermions. For simplicity, we assume that all components of $M_{Q}$ and $M_{L}$ are larger than the other mass parameters in the Yukawa terms, and integrate out the vector-like fermions. Then we obtain the effective Yukawa couplings for the SM fermions,

$$
\begin{aligned}
\mathcal{L}^{\text {Yukawa }} \simeq & -\sum_{i, j} \bar{q}_{L}^{i} \tilde{\phi}_{3} y_{u}^{i j} u_{R}^{j}-\sum_{i, j} \bar{q}_{L}^{i} \phi_{3} y_{d}^{i j} d_{R}^{j}-\sum_{i} \bar{\ell}_{L}^{i} \phi_{3} y_{e}^{i} e_{R}^{i} \\
& -\sum_{i, j} \bar{q}_{L}^{i} H_{1} \tilde{\phi}_{2}\left(Y_{Q 1} M_{Q}^{-1} Y_{2 u}\right)^{i j} u_{R}^{j}-\sum_{i, j} \bar{q}_{L}^{i} H_{1} \phi_{2}\left(Y_{Q 1} M_{Q}^{-1} Y_{2 d}\right)^{i j} d_{R}^{j} \\
& -\sum_{i, j} \bar{\ell}_{L}^{i} H_{1} \phi_{2}\left(Y_{L 1} M_{L}^{-1} Y_{2 e}\right)^{i j} e_{R}^{j} \\
& + \text { (h.c. }) .
\end{aligned}
$$

Now we can clearly see that this type of Yukawa couplings is categorized as the one in the type-III $2 \mathrm{HDM}$ with extra singlet scalar at low energy, after integrating out $W^{\prime}$ and $Z^{\prime}$ as we have done in section 3 . In the limit that $v_{1} \gg v_{2}$ and $h_{1}$ is extremely heavy, we lead the condition (iii): $\lambda_{4} \sim \lambda_{5} \sim \lambda_{6} \sim \lambda_{7} \sim 0$.

\subsection{Emergence of the type-II, -X, and -Y two Higgs doublet models with a discrete symmetry}

The type-II, -X, and -Y 2HDMs are also generated effectively from the setup in eq. (4.1) by controlling the Yukawa couplings. For example, if $y_{d}=y_{e}=Y_{2 u}=0$ are realized, the model behaves as the type-II $2 \mathrm{HDM}$ at low energy. A popular way to forbid unwanted terms is to assign a discrete symmetry. An illustrative $Z_{2}$ charge assignments for the three types are shown in table 4 . The $Z_{2}$ symmetry plays a role in controlling the Yukawa couplings of $\phi_{3}$, so that it is the same as the one in the ordinary 2HDMs. Besides, these assignments forbid $\kappa$ term which is corresponding to the soft mass term in the two-Higgs doublet model. However, $\kappa$ term is required to avoid spontaneous $Z_{2}$ symmetry breaking, and thus this $Z_{2}$ symmetry must be broken softly.

In the type-II, -X, and -Y 2HDMs which are emerged from this model, the CP-odd and the charged Higgs are automatically degenerated and the Higgs potential respects $\mathrm{CP}$ symmetry. However, since we introduce $Z_{2}$ symmetry to forbid unwanted Yukawa interactions, we can not address the origin of $Z_{2}$ symmetry as we did in the type-I $2 \mathrm{HDM}$ shown in section 3 . 


\begin{tabular}{|c|ccc|cccc|ccc|}
\hline$Z_{2}$ & $H_{1}$ & $H_{2}$ & $\phi_{3}$ & $Q_{L, R}$ & $L_{L, R}$ & $q_{L}$ & $\ell_{L}$ & $u_{R}$ & $d_{R}$ & $e_{R}$ \\
\hline type-II & \pm & \pm & - & \pm & \pm & + & + & - & + & + \\
type-X & \pm & \pm & - & \pm & \pm & + & + & - & - & + \\
type-Y & \pm & \pm & - & \pm & \pm & + & + & - & + & - \\
\hline
\end{tabular}

Table 4. Example of the $Z_{2}$ charge assignment for various two-Higgs doublet model.

\begin{tabular}{|c|cc|cc|cc|ccc|ccc|c|}
\hline $\mathrm{U}(1)$ & $q_{L}$ & $Q_{L}$ & $\ell_{L}$ & $L_{L}$ & $Q_{R}$ & $L_{R}$ & $u_{R}$ & $d_{R}$ & $e_{R}$ & $H_{1}$ & $\phi_{2}$ & $\phi_{3}$ & $S$ \\
\hline type-II & 0 & 0 & 0 & 0 & $x_{S}$ & $x_{S}$ & $x_{u}$ & $x_{d}$ & $x_{d}$ & $-x_{S}$ & $-x_{d}$ & $x_{u}$ & $x_{S}$ \\
\hline type-X & 0 & 0 & 0 & 0 & $x_{S}$ & $x_{S}$ & $x_{u}$ & $x_{u}$ & $x_{d}$ & $-x_{S}$ & $-x_{d}$ & $x_{u}$ & $x_{S}$ \\
\hline type-Y & 0 & 0 & 0 & 0 & $x_{S}$ & $x_{S}$ & $x_{u}$ & $x_{d}$ & $x_{u}$ & $-x_{S}$ & $-x_{d}$ & $x_{u}$ & $x_{S}$ \\
\hline
\end{tabular}

Table 5. Examples of $\mathrm{U}(1)$ charge assignments to forbid some Yukawa interaction terms. Here $x_{u} \neq x_{d}$. We take $x_{S}$ as $-\left(x_{u}+x_{d}\right)$ or $-\left(x_{u}+x_{d}\right) / 2$ to lead the soft $Z_{2}$ breaking term at low energy.

\subsection{Emergence of the type-II, $-\mathrm{X}$, and -Y two Higgs doublet models with a global symmetry}

There is another way to forbid unwanted terms without $Z_{2}$ symmetry. Instead of imposing $Z_{2}$ symmetry, we impose a global $\mathrm{U}(1)$ symmetry. In order to forbid some Yukawa interactions, the right-handed fermions, $H_{2}$, and $\phi_{3}$ have to be charged under this global symmetry. However, this means that the global symmetry is spontaneously broken by the Higgs VEVs, and predicts a NG boson whose decay constant is around the electroweak scale. In that case, the model is similar to the QCD axion model and already excluded.

To avoid the constraint, we have to extend our model, and introduce new gauge singlet scalar $S$. The charge assignment is given in table 5 . Here $x_{u} \neq x_{d}$ is required to forbid some Yukawa interaction terms. There are two choices for $x_{S}$. When $x_{S}=-\left(x_{u}+x_{d}\right)$ is satisfied, we can write down $\phi_{3}^{\dagger} H_{1} \phi_{2}$, while $x_{S}=-\left(x_{u}+x_{d}\right) / 2$ leads $\phi_{3}^{\dagger} H_{1} \phi_{2} S^{*}$. If $x_{S}$ does not satisfy both, the soft $Z_{2}$ breaking term is not emerged at low energy.

Since this model is similar to the DFSZ axion model [32], we expect that the VEV of $S$ is $\mathcal{O}\left(10^{11}\right) \mathrm{GeV}$. Then the VEV of $H_{1}$ should be also as large as the VEV of $S$ to reproduce the fermion masses. This naturally leads the decouplings of $\rho^{\mu}$ and $h_{1}$. In addition, we can solve the strong CP problem in the type-II and -Y cases if we choose $x_{S}=-\left(x_{u}+x_{d}\right) / 2$.

\section{Summary}

The structure of the SM gives some hints to the new physics behind the SM. One important prediction of the SM is very small flavor and $\mathrm{CP}$ violations, and another is small deviation of the $\rho$ parameter, which is realized by the custodial symmetry. These aspects strongly constrain the extensions of the SM.

The 2HDMs are widely discussed as candidates for BSMs. In the analysis of the 2HDMs, there are three features to realize the above conditions: (i) softly broken $Z_{2}$ symmetry, (ii) CP invariant Higgs potential, and (iii) degenerated mass spectra for the 
custodial symmetry. These three features play a crucial role in forbidding flavor violating Higgs interactions and avoiding large contribution to the $\rho$ parameter. Besides, they usually simplify setups and analyses. However, their origins are unclear, and they look artificial from a viewpoint of bottom-up approach.

In this paper, we have proposed a model with extended electroweak gauge symmetry, $\mathrm{SU}(2)_{0} \times \mathrm{SU}(2)_{1} \times \mathrm{U}(1)_{2}$, to explain the origins of the three conditions. We have shown that the low energy behavior of the model is well described by the type-I 2HDM with the three features. We also have discussed the extension of the models to derive other types of the 2HDM, and shown that the extended models can also solve the strong CP problem, imposing a global U(1) symmetry.

\section{Acknowledgments}

This work was supproted by JSPS KAKENHI Grant Number 16K17715.

Open Access. This article is distributed under the terms of the Creative Commons Attribution License (CC-BY 4.0), which permits any use, distribution and reproduction in any medium, provided the original author(s) and source are credited.

\section{References}

[1] ATLAS collaboration, Observation of a new particle in the search for the Standard Model Higgs boson with the ATLAS detector at the LHC, Phys. Lett. B 716 (2012) 1 [arXiv: 1207.7214] [INSPIRE].

[2] CMS collaboration, Observation of a new boson at a mass of $125 \mathrm{GeV}$ with the CMS experiment at the LHC, Phys. Lett. B 716 (2012) 30 [arXiv:1207.7235] [INSPIRE].

[3] A. Dedes and H.E. Haber, Can the Higgs sector contribute significantly to the muon anomalous magnetic moment?, JHEP 05 (2001) 006 [hep-ph/0102297] [INSPIRE].

[4] D. Chang, W.-F. Chang, C.-H. Chou and W.-Y. Keung, Large two loop contributions to g-2 from a generic pseudoscalar boson, Phys. Rev. D 63 (2001) 091301 [hep-ph/0009292] [INSPIRE].

[5] K.-M. Cheung, C.-H. Chou and O.C.W. Kong, Muon anomalous magnetic moment, two Higgs doublet model and supersymmetry, Phys. Rev. D 64 (2001) 111301 [hep-ph/0103183] [INSPIRE].

[6] K. Cheung and O.C.W. Kong, Can the two Higgs doublet model survive the constraint from the muon anomalous magnetic moment as suggested?, Phys. Rev. D 68 (2003) 053003 [hep-ph/0302111] [INSPIRE].

[7] Y.-L. Wu and Y.-F. Zhou, Muon anomalous magnetic moment in the Standard Model with two Higgs doublets, Phys. Rev. D 64 (2001) 115018 [hep-ph/0104056] [INSPIRE].

[8] J.F. Gunion, A light CP-odd Higgs boson and the muon anomalous magnetic moment, JHEP 08 (2009) 032 [arXiv:0808.2509] [INSPIRE].

[9] J. Cao, P. Wan, L. Wu and J.M. Yang, Lepton-specific two-Higgs doublet model: experimental constraints and implication on Higgs phenomenology, Phys. Rev. D 80 (2009) 071701 [arXiv:0909.5148] [INSPIRE]. 
[10] A. Broggio, E.J. Chun, M. Passera, K.M. Patel and S.K. Vempati, Limiting two-Higgs-doublet models, JHEP 11 (2014) 058 [arXiv:1409.3199] [INSPIRE].

[11] L. Wang and X.-F. Han, A light pseudoscalar of 2 HDM confronted with muon g-2 and experimental constraints, JHEP 05 (2015) 039 [arXiv:1412.4874] [INSPIRE].

[12] V. Ilisie, New Barr-Zee contributions to $(g-2)_{\mu}$ in two-Higgs-doublet models, JHEP 04 (2015) 077 [arXiv: 1502.04199] [INSPIRE].

[13] T. Abe, R. Sato and K. Yagyu, Lepton-specific two Higgs doublet model as a solution of muon g-2 anomaly, JHEP 07 (2015) 064 [arXiv: 1504.07059] [INSPIRE].

[14] T. Han, S.K. Kang and J. Sayre, Muon g-2 in the aligned two Higgs doublet model, JHEP 02 (2016) 097 [arXiv: 1511.05162] [INSPIRE].

[15] E.J. Chun and J. Kim, Leptonic precision test of leptophilic two-Higgs-doublet model, JHEP 07 (2016) 110 [arXiv:1605.06298] [INSPIRE].

[16] S.L. Glashow and S. Weinberg, Natural conservation laws for neutral currents, Phys. Rev. D 15 (1977) 1958 [INSPIRE].

[17] P.S. Bhupal Dev and A. Pilaftsis, Maximally symmetric two Higgs doublet model with natural Standard Model alignment, JHEP 12 (2014) 024 [Erratum ibid. 11 (2015) 147] [arXiv: 1408.3405] [INSPIRE].

[18] P. Ko, Y. Omura and C. Yu, A resolution of the flavor problem of two Higgs doublet models with an extra $\mathrm{U}(1)_{H}$ symmetry for Higgs flavor, Phys. Lett. B 717 (2012) 202 [arXiv: 1204 .4588] [INSPIRE].

[19] P. Ko, Y. Omura and C. Yu, Higgs phenomenology in type-I 2 HDM with $\mathrm{U}(1)_{H}$ Higgs gauge symmetry, JHEP 01 (2014) 016 [arXiv:1309.7156] [INSPIRE].

[20] P. Ko, Y. Omura and C. Yu, Higgs and dark matter physics in the type-II two-Higgs-doublet model inspired by $E_{6}$ GUT, JHEP 06 (2015) 034 [arXiv: 1502.00262] [INSPIRE].

[21] V.D. Barger, J.L. Hewett and R.J.N. Phillips, New constraints on the charged Higgs sector in two Higgs doublet models, Phys. Rev. D 41 (1990) 3421 [INSPIRE].

[22] Y. Grossman, Phenomenology of models with more than two Higgs doublets, Nucl. Phys. B 426 (1994) 355 [hep-ph/9401311] [INSPIRE].

[23] M. Aoki, S. Kanemura, K. Tsumura and K. Yagyu, Models of Yukawa interaction in the two Higgs doublet model and their collider phenomenology, Phys. Rev. D 80 (2009) 015017 [arXiv:0902.4665] [INSPIRE].

[24] J.-M. Gerard and M. Herquet, A twisted custodial symmetry in the two-Higgs-doublet model, Phys. Rev. Lett. 98 (2007) 251802 [hep-ph/0703051] [INSPIRE].

[25] Particle Data Group collaboration, K.A. Olive et al., Review of particle physics, Chin. Phys. C 38 (2014) 090001 [InSPIRE].

[26] N.G. Deshpande and E. Ma, Pattern of symmetry breaking with two Higgs doublets, Phys. Rev. D 18 (1978) 2574 [inSPIRE].

[27] R. Barbieri, L.J. Hall and V.S. Rychkov, Improved naturalness with a heavy Higgs: an alternative road to LHC physics, Phys. Rev. D 74 (2006) 015007 [hep-ph/0603188] [INSPIRE].

[28] T. Abe and R. Kitano, Phenomenology of partially composite Standard Model, Phys. Rev. D 88 (2013) 015019 [arXiv: 1305.2047] [INSPIRE]. 
[29] E. Cerveró and J.-M. Gérard, Minimal violation of flavour and custodial symmetries in a vectophobic two-Higgs-doublet-model, Phys. Lett. B 712 (2012) 255 [arXiv:1202.1973] [INSPIRE].

[30] R. Sekhar Chivukula, N.D. Christensen, B. Coleppa and E.H. Simmons, The top triangle moose: combining higgsless and topcolor mechanisms for mass generation, Phys. Rev. D 80 (2009) 035011 [arXiv:0906.5567] [InSPIRE].

[31] M. Buen-Abad, A.G. Cohen and M. Schmaltz, A model for the LHC diboson excess, JHEP 05 (2016) 111 [arXiv:1604.03578] [INSPIRE].

[32] M. Dine, W. Fischler and M. Srednicki, A simple solution to the strong CP problem with a harmless axion, Phys. Lett. B 104 (1981) 199 [INSPIRE]. 